\title{
The non-specific and sex-differential effects of vaccines
}

\section{Peter Aaby, Christine Stabell Benn, Katie L. Flanagan, Sabra L. Klein, Tobias R. Kollmann (D), David J. Lynn (D) and Frank Shann}

Abstract | The textbook view of vaccination is that it functions to induce immune memory of the specific pathogen components of the vaccine, leading to a quantitatively and qualitatively better response if the host is exposed to infection with the same pathogen. However, evidence accumulated over the past few decades increasingly suggests that vaccines can also have non-specific effects on unrelated infections and diseases, with important implications for childhood mortality particularly in low-income settings. Furthermore, many of these non-specific effects, as well as the pathogen-specific effects, of vaccines show differences between the sexes. Here, members of the Optimmunize consortium discuss the evidence for and potential mechanisms of non-specific and sex-differential effects of vaccines, as well as their potential policy implications. Given that the non-specific effects of some vaccines are now being tested for their ability to protect against COVID-19, the authors also comment on the broader implications of these trials.

For which vaccines and in which clinical contexts are non-specific effects of vaccines important? Are there differences between live and non-live vaccines? Can effects be both beneficial and harmful?

David J. Lynn. In my opinion, there is now substantial evidence that several different vaccines can have non-specific effects (NSEs; also known as heterologous effects) on immune responses, morbidity and mortality. A 2016 systematic review sponsored by the World Health Organization (WHO) concluded that the bacillus Calmette-Guérin (BCG) vaccine, diphtheria-tetanus-whole cell pertussis $(\mathrm{DTPw})$ vaccine and measles vaccine were associated with effects on mortality that were "more than would be expected through their effects on the diseases they prevent"

Katie L. Flanagan and Frank Shann. Theoretically, any vaccine can have NSEs because microbial antigens in vaccines stimulate an early innate immune response through pattern recognition receptors on immune cells. Some of the most compelling evidence for NSEs comes from randomized and observational studies in low-income settings ${ }^{2,3}$, where live vaccines have been shown to have beneficial NSEs on all-cause childhood mortality but non-live vaccines might have detrimental NSEs. Combinations of live and non-live vaccines given at the same time have variable effects, with the NSEs being determined largely by the most recent vaccine administered ${ }^{1,4}$.

Peter Aaby and Christine Stabell Benn. So far, all vaccines tested in epidemiological studies have shown important NSEs on child survival in low-income countries. Observations support a pattern whereby live vaccines (such as smallpox vaccine, $B C G$ vaccine, measles vaccine and oral polio vaccine (OPV)) increase resistance to vaccine-unrelated infections, mainly pneumonia and sepsis, and therefore reduce overall mortality more than vaccine-targeted infections. Hence, these live vaccines have a double benefit in that they prevent both target and non-target infections ${ }^{4}$. By contrast, non-live vaccines (such as DTP vaccine, the pentavalent vaccine for DTP, hepatitis B virus (HBV) would be expected from preventing the and Haemophilus influenzae type b, inactivated polio vaccine, single HBV vaccine, the RTS,S/AS01 malaria vaccine, and the H1N1 influenza vaccine) seem to increase susceptibility to vaccine-unrelated infections, particularly in females ${ }^{4}$. Hence, non-live vaccines may have beneficial effects in preventing the target infection but negative effects by enhancing susceptibility to non-target infections. In epidemiological studies, the negative effects seem to be more pronounced than the beneficial effects, with the net effect being increased overall mortality for females. Fortunately, the most recent vaccine to be administered has the strongest NSEs, and so the negative effects of non-live vaccines can be at least partly abrogated by providing a live vaccine after the non-live vaccine.

K.L.F. and F. S. Evidence from multiple observational studies suggests that non-live vaccines, such as DTP vaccine, may increase all-cause mortality, especially in girls, because there is an increased number of deaths from pneumonia and sepsis that outweighs the reduction in deaths from diphtheria, tetanus and pertussis ${ }^{5}$. In a large randomized trial, the non-live RTS,S/AS01 malaria vaccine doubled all-cause mortality in girls in Africa ${ }^{6}$.

Because of the potential for large effects on all-cause mortality, the NSEs of vaccines are most important in children younger than 5 years in high-mortality countries, where the vaccine schedule is for children to be immunized with live BCG vaccine and OPV at birth, non-live DTP vaccine at 6,10 and 14 weeks of age and live measles vaccine at 9 months of age. DTP vaccine is often given with non-live inactivated polio vaccine, HBV vaccine, $H$. influenzae type b vaccine and pneumococcal conjugate vaccine, as well as live OPV and rotavirus vaccine.

D.J.L. More than ten studies have found that non-live vaccines (such as DTPw vaccine) are associated with increased all-cause mortality, particularly in girls . It is important to note that the DTP vaccine is highly effective against the targeted diseases and, so far, the studies reporting deleterious NSEs have been observational and have been assessed to be at a high risk of bias. Perhaps understandably, in an age 
of increasing vaccine hesitancy, many in the research community are resistant to considering that such deleterious NSEs could exist. However, if they do, there are potentially relatively easy solutions to mitigate these effects: substantial evidence is mounting that any deleterious effects of non-live vaccines can be mitigated by changing vaccine schedules so that a live vaccine is administered last ${ }^{8}$.

Have there been similar observations in low-income and high-income settings?

K.L.F. and F.S. NSEs of vaccines have been described in both low-income and high-income settings. In low-income settings, as discussed already, much of the evidence relates to changes in all-cause mortality ${ }^{2,3}$, although morbidity effects have also been widely described. In high-income countries, several studies from Europe and the USA suggest that admission to hospital for unrelated infections may be reduced by live measles vaccine and BCG vaccine but increased by non-live DTP vaccine ${ }^{9,10}$. Evidence from high-income countries also indicates that BCG vaccination may reduce the severity of allergy ${ }^{11}$, malignancy ${ }^{12-14}$, diabetes $^{15}$ and Alzheimer disease ${ }^{16,17}$.

D.J.L. Most studies so far have been conducted in infants in low-income countries, although there have also been some studies in infants in high-income countries $^{18}$. One study in Spain found that hospitalization rates owing to respiratory infections, not attributable to tuberculosis, were significantly lower in BCG-vaccinated children $^{18}$. The hospitalization rate owing to sepsis in infants younger than 1 year was also significantly lower after BCG vaccination. Furthermore, three randomized controlled trials in low-birthweight neonates in Guinea-Bissau reported beneficial NSEs of BCG immunization ${ }^{19}$. However, another study, in Denmark, found that BCG vaccination at birth did not reduce the risk of hospitalization for somatic acquired disease (in other words, disease excluding injuries ${ }^{20}$. It is not entirely clear why this study failed to detect a beneficial NSE of BCG vaccine, but it may relate to this measure of disease not being specific enough, differences in exposure between countries or differences in genetics or maternal immunity.

There have also been a small number of studies in adults in high-income countries. For example, a randomized placebo-controlled trial found that BCG vaccination protected Dutch adult volunteers

\section{The contributors}

Peter Aaby was trained as an anthropologist but has built a large health surveillance system in Guinea-Bissau since 1978, focusing on the high levels of child mortality there. Crowding and intensive exposure to measles were key determinants of child mortality. This led to vaccine research and the discovery of the non-specific effects of measles vaccine.

Christine Stabell Benn is a professor in global health at the University of Southern Denmark. She conducts epidemiological and immunological studies of vaccines and vitamin A, with a focus on their real-life effects on overall health in Africa and Denmark. She formulated the hypothesis that these health interventions with immunomodulatory effects interact, often in a sex-differential manner.

Katie L. Flanagan is Director of Infectious Diseases for north/north-west Tasmania, an adjunct professor at the University of Tasmania and RMIT University and an adjunct associate professor at Monash University. She is Honorary Secretary of the Australasian Society for Infectious Diseases (ASID), Chair of the ASID Vaccination Special Interest Group and a member of the Australian Technical Advisory Group on Immunisation. Her current research focuses on using systems vaccinology to study the sex-differential and non-targeted effects of vaccines.

Sabra L. Klein is a professor of molecular microbiology and immunology at the Johns Hopkins Bloomberg School of Public Health, Baltimore, USA. She is an expert on sex and gender differences in immune responses and susceptibility to infection. She is the immediate past president of the Organization for the Study of Sex Differences, a principal investigator of the Johns Hopkins Specialized Center for Research Excellence in sex and age differences in immunity to influenza and a co-director of the Johns Hopkins Center for Women's Health, Sex, and Gender Research.

Tobias R. Kollmann is a paediatric infectious disease clinician and systems vaccinologist at Telethon Kids Institute and Perth Children's Hospital in Perth, Australia. His expertise centres on newborn infectious diseases, immune ontogeny and early-life vaccine responses, using cutting-edge technology and analytics to extract the most information out of the typically small biological samples obtainable in early life.

David J. Lynn is Director of the Computational and Systems Biology Program and an EMBL Australia group leader at the South Australian Health and Medical Research Institute. He is also a professor at the Flinders University College of Medicine and Public Health. He leads a research programme in systems immunology, investigating how pathogenic and commensal microorganisms modulate the immune system in different contexts, including vaccination.

Frank Shann worked as a paediatrician in Papua New Guinea and then for 20 years was Director of Intensive Care at the Royal Children's Hospital in Melbourne, Australia. He is a professorial fellow in the Department of Paediatrics, University of Melbourne, engaged in research on the non-specific effects of vaccines.

against experimental infection 1 month later with an attenuated yellow fever virus vaccine strain $^{21}$, and BCG vaccination once a month for three consecutive months was found to significantly reduce acute upper respiratory tract infection in older individuals ${ }^{22}$.

P.A. and C.S.B. We have been able to repeat the original observations from Guinea-Bissau of NSEs of vaccines in other low-income countries (such as Bangladesh, the Gambia, Ghana, India, Kenya, Malawi, Senegal and Sudan ${ }^{23}$ and also in a high-income setting in Denmark. In Denmark, child mortality is not a suitable outcome and we have therefore examined NSEs by testing the effect of vaccines on hospital admissions of children for non-target infections? Furthermore, in historical studies of BCG and smallpox vaccines, we have been able to find associations between these live vaccines and decreased adult mortality ${ }^{24}$. The observations from other low-income countries seem to confirm the differential patterns of beneficial and harmful NSEs for live vaccines and non-live vaccines, respectively ${ }^{4,23}$. In Denmark, it has been easier to test the potential beneficial NSEs of live vaccines; so far, we have found beneficial NSEs of measles-mumps-rubella (MMR) vaccine, OPV, BCG vaccine and smallpox vaccine ${ }^{9,24}$. The MMR vaccine has been associated with decreased hospital admissions for respiratory infections also in the USA, Italy and the Netherlands ${ }^{4}$. The data from Denmark also indicate that giving the non-live DTP vaccine after the MMR vaccine may increase the risk of hospital admission by cancelling out the positive effect of the MMR vaccine ${ }^{9}$.

Can you comment on the differences between males and females for these non-specific effects, as well as for vaccine responses generally?

Sabra L. Klein Let us begin with the vaccine-specific responses. Females of diverse ages typically develop a greater 
antibody response (which is a primary correlate of protection) and report more adverse reactions to vaccines than do males $^{25}$. For example, after vaccination against influenza, yellow fever, rubella, measles, mumps, hepatitis A, hepatitis B, herpes simplex type 2 , rabies, smallpox and dengue viruses, protective antibody responses are twice as large in adult females as in adult males ${ }^{25}$.

Regarding sex differences in NSEs, many studies have documented detrimental, female-biased effects. There is a growing body of literature showing that infant girls have increased mortality after receiving certain vaccines. For example, in the 1980s, when the high-titre measles vaccine (HTMV) was introduced to prevent measles infection in children younger than 9 months, there was a twofold increase in all-cause mortality in girls, but no increase in boys, in Guinea-Bissau ${ }^{26}$. It was subsequently determined that the increased mortality occurred only among girls who had received non-live DTP vaccine after HTMV, and not among girls who had received HTMV after their last dose of DTP vaccine ${ }^{27}$. The interaction between HTMV and DTP vaccine may have caused NSEs on all-cause mortality in girls, but not boys. Evidence from multiple studies of non-live vaccines, including DTP vaccine and the inactivated polio vaccine, shows that they have greater detrimental NSEs for girls than for boys ${ }^{27,28}$. More recently, increased female mortality after receipt of the RTS,S/AS01 malaria vaccine was reported in post hoc analyses of data ${ }^{6}$. The WHO has speculated that the increased mortality in girls was 'largely due to the low female mortality in the control arm' and 'could be due to chance', despite the $P$ value of 0.0006 for girls, and a mortality after RTS,S/AS01 vaccination of $2.4 \%$ in girls compared with $1.8 \%$ in boys $^{29}$. This vaccine has been rolled out into routine vaccination schedules without further studies to determine whether the RTS,S/AS01 vaccine does indeed increase mortality in girls. In keeping with NSEs disproportionately affecting females, data pertaining to the NSEs of BCG vaccine show that the beneficial effects on all-cause mortality are greater for girls than for boys younger than 5 years ${ }^{3}$.

K.L.F. and F.S. Most studies have not analysed vaccine responses for sex differences but, when they have, sex differences in immunogenicity have been found for almost every licensed vaccine $^{30,31}$. Generally, females have greater antibody responses to immunization, but sex differences in terms of cellular responses to immunization have rarely been investigated and are inconsistent ${ }^{30}$. Females also have more adverse events following immunization ${ }^{25}$, perhaps because of biological sex differences, although reporting differences (a gender effect) may also have a role. Females are generally more susceptible than males to the NSEs of vaccines; for example, females benefit more from the beneficial NSEs of measles vaccine ${ }^{32}$ but experience greater adverse NSEs following immunization with DTP vaccine vi, $^{3,7}$.

P.A. and C.S.B. One of the first major discoveries in relation to NSEs was that HTMV was associated with twofold increased female mortality in Guinea-Bissau and Senegal. Even though HTMV protected against measles, the negative NSEs meant that there was an overall increase in female mortality ${ }^{27}$. Potential sex-differential effects of vaccines have therefore been a focus in the study of NSEs. In West Africa, where there were no major sex differences in terms of treatment or mortality in the prevaccine era, it has been a strong indication of NSEs when vaccines have sex-differential effects on overall mortality $^{4,23}$. The live measles, BCG and smallpox vaccines have had stronger beneficial NSEs for females than for males. By contrast, the six non-live vaccines tested (DTP vaccine, the pentavalent vaccine for DTP, HBV and $H$. influenzae type b, inactivated polio vaccine, single HBV vaccine, the RTS,S/AS01 malaria vaccine and the H1N1 influenza vaccine) have all been associated with higher female mortality than male mortality ${ }^{4}$. This is a worrying finding, and nobody has been able to suggest a bias that might explain it. The effects are not trivial - data indicate that on the scale of Africa, hundreds of thousands of females per year may die owing to the negative NSEs of non-live vaccines.

\section{What do we know and not know about the mechanism of these non-specific and sex-differential effects?}

D.J.L. The mechanisms behind the NSEs of vaccines are incompletely understood but are likely to involve a combination of different effects on the innate and adaptive immune responses, heterologous $\mathrm{T}$ cell responses and influences on responses to other subsequent immunizations. One mechanism that we now know quite a lot about is trained immunity - a programme of innate immune memory that can be induced by vaccination, leading to the epigenetic and metabolic reprogramming of innate immune cells such as monocytes and natural killer cells ${ }^{14}$. However, most of what we know regarding trained immunity comes from studies of BCG vaccine and we know far less about whether other vaccines also induce trained immunity and, if so, whether they do so differently to $B C G$ vaccine. Further research is therefore urgently needed to understand the potential immunological mechanisms that could explain the differential NSEs of live and non-live vaccines. This is an area of research that my laboratory is investigating.

P.A. and C.S.B. There are at least two mechanisms that have been documented: trained immunity and emergency granulopoesis (see the response from Tobias R. Kollmann $)^{33,34}$. In addition, heterologous $\mathrm{T}$ cell reactivity may also have a role ${ }^{33}$. One very important new observation from epidemiological studies is that the beneficial NSEs of live vaccines such as measles vaccine and BCG vaccine become more pronounced if the vaccine is administered in the presence of pre-existing immunity ${ }^{4,35}$, for example, if the mother was previously vaccinated with the same vaccine (vertical boosting) or if the child is vaccinated for a second time with a live vaccine (horizontal boosting) ${ }^{36}$. We are currently exploring in mechanistic studies why boosting results in amplification of the beneficial NSEs for the child.

Tobias R. Kollmann. We recently discovered one of the mechanisms by which BCG vaccine can protect newborns from infection ${ }^{34}$. Within hours of administration, BCG vaccine induces production of the growth factor granulocyte colony-stimulating factor (G-CSF), which in turn activates a process known as emergency granulopoiesis that increases the production of neutrophils ready to fight infectious threats. The kinetics of this rapid response to BCG vaccine fit perfectly with the epidemiological observations that BCG vaccine can protect newborns within just days of administration ${ }^{19,37}$.

K.L.F. and F.S. Although the induction of vaccine-specific antibodies is generally considered to be a reliable surrogate marker of a protective vaccine response, we still do not understand the mechanisms for the specific effects of many vaccines. For example, we do not know how BCG vaccine protects against tuberculosis. However, we are beginning to understand the immunological mechanisms accounting for the NSEs of some vaccines. Human studies have shown that BCG vaccine enhances innate immunity 
within days after immunization by epigenetic reprogramming that leads to trained immunity ${ }^{14}$. By contrast, immunization with DTP vaccine has been shown to suppress innate immunity and induce T cell anergy in female infants but not male infants, and this could explain how DTP vaccine might increase susceptibility to infections in females $^{38}$. Theoretical biological mechanisms for sex differences in the NSEs include the opposing immunological effects of male and female sex hormones, the multiple X-linked (and some Y-linked) immune response genes and microRNAs, and sex differences in the microbiota, although causal links have not yet been confirmed ${ }^{30,31}$.

S.L.K. So far, we do not know the mechanisms of sex differences in NSEs. We do, however, have data pertaining to sex differences in vaccine-specific immunity. Following vaccination with whole inactivated influenza virus, influenza trivalent inactivated vaccine or influenza quadrivalent inactivated vaccine, adult female mice generate greater quantity and quality of influenza-specific antibodies than do adult male mice ${ }^{39-41}$. Antibodies derived from vaccinated female mice are better at protecting naive mice (both male and female) than are antibodies from vaccinated male mice, and this protection is associated with increased antibody specificity and avidity for the H1N1 influenza virus ${ }^{41}$. The Toll-like receptor 7 gene ( $T l r 7$ ) is encoded on the $\mathrm{X}$ chromosome and is expressed at a higher level by B cells from vaccinated female mice than by B cells from vaccinated male mice, which is associated with reduced DNA methylation in the Tlr7 promoter region in female mice ${ }^{41}$. Data from humans and mice further show that the female bias of antibody responses to influenza vaccination is associated with circulating oestradiol levels, such that greater concentrations of oestradiol result in greater production of antibody to the vaccine antigen ${ }^{42}$. Taken together, both the expression of X-linked genes and increased levels of oestradiol are associated with improved vaccine-induced immunity in females. We now need to determine how to use this information to improve vaccine-induced immunity in males (for example, through the use of TLR7 adjuvants).

\section{Should we consider changes to vaccine types, doses or schedules on the basis of} these effects?

D.J.L. As discussed already, live vaccines have generally been associated with beneficial NSEs and non-live vaccines have been associated with potentially deleterious NSEs in some observational studies. There is also evidence that any deleterious effects of non-live vaccines can be mitigated by changing vaccine schedules such that a live vaccine is administered last ${ }^{8}$. In my opinion, however, it is too early to recommend any changes to schedules until further high-quality randomized controlled trials are conducted to assess the effects of such changes. Given the mounting data, these studies need to be urgently supported and conducted.

P.A. and C.S.B. We believe that there are several immediate considerations as a result of these NSEs. First, all children in Africa should receive the BCG vaccine at birth. This has been shown to reduce neonatal mortality by more than one-third ${ }^{4}$, but currently less than $50 \%$ of children in Africa receive $B C G$ vaccine in the first month of life. We should promote the use of BCG vaccine as a non-specific vaccine to boost the baby's immune system. Second, we should roll back the plan to phase out OPV. Vaccination campaigns with OPV have had a major role in reducing child mortality in low-income countries, with vaccination of only 68 children being needed to save the life of 1 child $^{43}$. Hence, the benefits of OPV outweigh the minor risk of vaccine-derived polio infection. Third, we should ensure that children are given a live vaccine shortly after receiving non-live vaccines. For example, all studies show that the administration of DTP vaccine after measles vaccine is associated with higher female mortality than is administration of measles vaccine after DTP vaccine $^{23}$. This might also explain why live HTMV had been associated with increased female mortality: HTMV was given so early in life that most children received DTP vaccine after $\mathrm{HTMV}^{27}$.

F.S. There is already far greater evidence of harm from the NSEs of DTP vaccine in low-income countries than there was of harm from the rotavirus vaccine RotaShield when its use was suspended in the USA in 1999 after only 15 cases of intussusception. However, it is essential that we continue to immunize children against diphtheria, tetanus and pertussis. In high-mortality countries where BCG vaccine is given routinely at birth, DTP vaccine and other non-live vaccines could be given safely at 6,10 and 14 weeks of age if a second dose of live BCG vaccine were to be given at the same time as the last priming dose of DTP vaccine at 14 weeks. After BCG vaccine administration at 14 weeks of age, I believe that no non-live vaccines should then be given to children younger than 5 years in high-mortality countries without robust evidence of safety from randomized trials. It has been estimated that this change in policy could save approximately 1 million lives every year ${ }^{8}$.

What more do we need to know for these non-specific and sex-differential effects to be more widely accepted and the implications to be more widely considered?

D.J.L. There is still much to be understood regarding the NSEs and sex-differential effects of vaccines. We currently do not fully understand the mechanistic basis as to why non-live vaccines may be associated with deleterious NSEs, whereas live vaccines are associated with beneficial NSEs. We also do not understand the mechanisms through which changes to the order in which vaccines are administered may alter these NSEs. We also urgently need large, well-funded and international randomized controlled trials to confirm the data suggesting that non-live vaccines are associated with detrimental NSEs and to support any changes to vaccine schedules. Importantly, the international vaccine community should not be afraid to consider that there may be both beneficial and detrimental NSEs of certain vaccines. We are acutely aware of the potential for research in this area to be unintentionally or wilfully misinterpreted by individuals with antivaccine agendas. Understanding the NSEs of vaccines may result in exciting new beneficial interventions for unrelated infectious diseases and for non-infectious diseases such as allergy, asthma and even diabetes, while at the same time allowing us to mitigate against any potential risks.

P.A. and C.S.B. The argument can be split in two. First, do vaccines have only specific effects? The answer is clearly no! There is overwhelming evidence that vaccines affect the immune system more generally. Epidemiologically, we see effects of vaccines that cannot be explained by their specific effects $^{4}$. Immunologically, we see that vaccines leave long-lasting imprints on the immune system that alter responses to heterologous challenges ${ }^{33,34}$. Second, can we predict the NSEs of any vaccine? We have seen repeatable patterns - for example, that live vaccines are associated with beneficial NSEs and non-live vaccines are associated with negative NSEs for females ${ }^{4}$ - that made it possible to predict that the RTS,S/AS01 vaccine would have negative NSEs for 
females ${ }^{4}$. This prediction indicates that we are on the right track with regard to our current thinking about NSEs. To move forward, we need to continue to make predictions and test them to refine our knowledge of the principles of NSEs.

F.S. We urgently need a trial in which children are randomized to receive or not receive a second dose of BCG vaccine with the last priming dose of DTP vaccine at 14 weeks of age in a high-mortality country, with a comparison of mortality from 14 weeks to 9 months of age (when measles vaccine is given $)^{8}$. The same cohort of children should then be randomized again in the second year of life to receive or not receive the booster dose of DTP vaccine (which is recommended by the WHO but is not currently given in many countries, especially in countries in Africa, where it is not included in national vaccination schedules), with comparison of mortality from randomization to 3 years of age ${ }^{8}$. This would tell us whether DTP vaccine increases all-cause mortality and whether a second dose of BCG vaccine can ameliorate any harmful NSEs of DTP vaccine.

K.L.F. and F.S. We recommend using a systems vaccinology approach to elucidate the mechanisms of the NSEs of vaccines. Transcriptomics, epigenomics, proteomics and metabolomics will reveal the complex immunological pathways that are influenced by immunization ${ }^{44}$. This approach might identify the immunological correlates of both the beneficial NSEs and the harmful NSEs of vaccines and could be used to improve the design of future vaccines, adjuvants and immunomodulatory strategies.

S.L.K. We need more information on the mechanisms of NSEs. To address mechanisms, we need models, both animals and primary cell culture systems, that can be used to test the NSEs in both males and females. We also need more research into the sex differences in vaccine-specific immunity, with more data from diverse vaccine platforms. At the Optimmunize meeting held in February 2020, data on sex-differential effects were presented for vaccines that protect against influenza virus, rabies virus and even simian immunodeficiency virus.

T.R.K. The real hurdle before us is not so much the lack of knowledge but more so the willingness of the 'gatekeepers' of vaccination programmes around the world to even consider that vaccines might impact the host beyond the expected pathogen-specific effects. For example, the rapidity of BCG-mediated protection from newborn infection has been used to call into question the biological plausibility of NSEs of vaccines, but we now know that vaccines can have such rapid effects, for example, through emergency granulopoiesis ${ }^{34}$. The protective power of the immune system rests - at least in part - on its ability to take into account a multitude of signals, including non-specific, pathogen-agnostic effects, and to do so very, very fast.

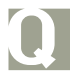
At the time of going to press, with the COVID-19 pandemic dominating headlines around the globe, how do you think these non-specific and/or sex-differential effects of vaccines might be relevant to efforts to combat COVID-19?

D.J.L. The best hope for tackling the COVID-19 pandemic is a specific vaccine that provides direct protection against the virus. Efforts to develop such a vaccine are proceeding around the world at a breakneck pace, but an effective vaccine is still likely to be at least 12 months away. In the interim, several groups around the world have hypothesized that certain existing live vaccines, such as BCG vaccine, may provide non-specific protection against COVID-19 or reduce the severity of its symptoms. As discussed here, BCG vaccine is associated with non-specific protection against respiratory and viral infections in other contexts, and can non-specifically boost innate immune responses. Several epidemiological studies have also emerged as preprints suggesting that countries with routine BCG immunization programmes have lower case fatality rates for COVID-19 than countries that do not routinely use BCG vaccine ${ }^{45-47}$. However, it is important to note that such studies provide correlations not causation, and it is inherently difficult to account for all potential confounders, particularly given that the case statistics in each country are still highly uncertain. Nevertheless, several randomized controlled trials are now under way around the world to definitively assess whether BCG vaccine can provide non-specific protection against COVID-19. These studies include the BRACE trial ('BCG vaccination to reduce the impact of COVID-19 in Australian healthcare workers following coronavirus exposure'), which my laboratory is helping to roll out in South Australia. In this trial, thousands of health-care workers at hospitals around Australia are being randomized to receive $B C G$ vaccine or not receive it, and we will assess whether those in the BCG vaccination arm are better protected against or have reduced severity of COVID-19. The world will be watching the outcome of these trials with significant interest, not only for an effect against COVID-19 but also as a demonstration of the potential importance of NSEs of vaccines more generally. Until then, however, BCG vaccine should be used only for its intended purpose of protecting infants globally against tuberculosis.

K.L.F. and F.S. Yellow fever virus, murine Mengo virus (also known as encephalomyocarditis virus) and severe acute respiratory syndrome coronavirus 2 (SARS-CoV-2) are all single-stranded positive-sense RNA viruses. As the BCG vaccine has been shown to reduce the severity of infection with yellow fever virus and Mengo virus ${ }^{21,48}$, this suggests that administration of BCG vaccine might reduce the severity of COVID-19 (REF. ${ }^{21}$ ). However, there is no evidence as yet of a protective effect from administration of a live vaccine during an infection with SARS-CoV-2, and it is unlikely that BCG vaccine given many decades ago will influence the response to SARS-CoV-2 now. If BCG vaccine is used, we think that it should be given with or after any non-live vaccine such as influenza vaccine ${ }^{2,4}$. If other live vaccines such as measles vaccine or OPV are used, they should be given at least 4 weeks after any non-live vaccine ${ }^{32}$.

T.R.K. Given that there is no other form of intervention currently available, it would be foolish not to at least consider whether the fast and broadly protective NSEs of live vaccines (such as BCG vaccine, OPV and measles vaccine) might provide protection from severe COVID-19 (REF. ${ }^{49}$ ). But to be clear, there is currently no evidence I know of that shows that any of these live vaccines are in fact reducing the risk of either infection with SARS-CoV-2 or the resulting disease, COVID-19. So yes, they should be tested, but with the clear understanding that we currently simply do not know.

This is in fact going on already, as BCG vaccination is being tested in randomized clinical trials as a prophylactic vaccine in health-care workers in Australia (the BRACE trial mentioned earlier) and the Netherlands, and OPV is also being considered as an intervention. An additional advantage of this approach rests on the solid safety record of these live vaccines compared with many of the other interventions that are currently being considered for COVID-19, such as anti-HIV drugs. Importantly, even if a vaccine for COVID-19 were available 
soon, this vaccine would have no record of either efficacy or safety - in other words, compared with the NSEs of our existing live vaccines, there is nothing we have available to use now that is as promising, as safe and as cheap.

One additional problematic issue to be aware of in this context is the already tenuous supply of BCG vaccine for newborns in the world, in whom it saves hundreds of thousands of lives every year through its pathogen-specific effects (targeting tuberculosis) as well as NSEs. If the story of hydroxycholoroquine as an unproven therapy for COVID-19 is any measure to anticipate what might happen, then any notion of BCG vaccine possibly protecting against COVID-19 might lead to a rush to buy up all existing $B C G$ vaccine by higher-income countries. Unfortunately, this is already starting to happen. If this continues, without any internationally coordinated effort to rapidly expand production of BCG vaccine (which is necessary no matter what), then our response to SARS-CoV-2 could be responsible for many deaths in newborns from tuberculosis or sepsis ${ }^{37}$. This would be a severe violation of social justice.

K.L.F. and F.S. It is important that increased use of BCG vaccine in an attempt to reduce the severity of COVID-19 in rich countries does not cause a shortage of BCG vaccine in high-mortality, lower-income countries, where it is urgently needed to protect young children against tuberculosis ${ }^{49}$.

S.L.K. With ongoing studies around the world, including by members of the Optimmunize consortium, evaluating the possible beneficial NSEs of BCG vaccine on outcomes of COVID-19, more consideration should be given to the sex of the trial participants as it may be that the beneficial NSEs are greater for females than for males. With the reports of male-biased severe outcomes of COVID-19 $\left(R^{2} F^{50}\right)$, it is pertinent that we identify treatments that work equally well in males as they do in females. As we evaluate the efficacy of candidate COVID-19 vaccines, sex-differential antibody responses and protection should be considered in all vaccine trials. In a not-yet peer-reviewed study of 331 patients with confirmed SARS-CoV-2 infection in Wuhan, China, anti-SARS-CoV-2 IgG responses were measured and compared among patients with clinically diagnosed mild disease or severe disease ${ }^{51}$. Among patients with mild COVID-19, anti-SARS-CoV-2 IgG titres were similar between the sexes. By contrast, among patients with severe disease, females had greater antibody responses than males, with production of antibodies at earlier phases of disease, which suggests one possible immunological mechanism by which women might recover better from COVID-19 than men ${ }^{51}$.

P.A. and C.S.B. We should also consider that the COVID-19 pandemic is likely to lead to delayed vaccinations and lower vaccine coverage worldwide. Missing the specific effects and particularly the NSEs of BCG vaccine, OPV and measles vaccine could easily result in far more deaths than the deaths caused by COVID-19. The COVID-19 pandemic is exposing our total reliance on the 'one-vaccine-to-one-disease' concept of health. The beneficial NSEs of some live vaccines clearly indicate that it is possible to train immunity to develop stronger resistance to unrelated infections. How to develop this capacity should become a major research priority. In the future, we will need a one-vaccine-to-many-diseases concept of health.

Peter Aaby ${ }^{1 凶}$, Christine Stabell Benn $n^{2,3 凶}$, Katie L. Flanagan ${ }^{4,5,6}{ }^{凶}$, Sabra L. Klein ${ }^{7 凶}$, Tobias R. Kollmann (D) ${ }^{8 凶}$, David J. Lynn (iD) ${ }^{9,10 凶}$ and Frank Shann ${ }^{11 凶}$

'Bandim Health Project, Bissau, Guinea-Bissau. ${ }^{2}$ Bandim Health Project, OPEN, Department of Clinical Research, Odense University Hospital, Odense, Denmark.

${ }^{3}$ Danish Institute for Advanced Study, University of Southern Denmark, Odense, Denmark.

${ }^{4}$ University of Tasmania, Hobart, TAS, Australia.

${ }^{5}$ RMIT University, Melbourne, VIC, Australia.

${ }^{6}$ Monash University, Melbourne, VIC, Australia.

${ }^{7}$ W. Harry Feinstone Department of Molecular Microbiology and Immunology, Johns Hopkins Bloomberg School of Public Health, Baltimore, MD, USA.

${ }^{8}$ Telethon Kids Institute, Perth, WA, Australia.

${ }^{9}$ South Australian Health and Medical Research Institute, Adelaide, SA, Australia.

${ }^{10}$ Flinders University, Adelaide, SA, Australia.

"Department of Paediatrics, University of Melbourne, Melbourne, VIC, Australia.

凶e-mail: p.aaby@bandim.org; cbenn@health.sdu.dk; katieflanagan@ths.tas.gov.au; sklein2@jhu.edu; tkollm@mac.com; david.lynn@sahmri.com;

fshann@unimelb.edu.au

https://doi.org/10.1038/s41577-020-0338-x

Published online 27 May 2020

1. Higgins, J. P. et al. Association of BCG, DTP, and measles containing vaccines with childhood mortality: systematic review. BMJ 355, i5170 (2016)

2. Shann, F. Nonspecific effects of vaccines and the reduction of mortality in children. Clin. Ther. 35 109-114 (2013).

3. Aaby, P., Kollmann, T. R. \& Benn, C. S. Nonspecific effects of neonatal and infant vaccination: public-health, immunological and conceptual challenges. Nat. Immunol. 15, 895-899 (2014).
4. Benn, C. S., Fisker, A. B., Rieckmann, A., Sørup, S $\&$ Aaby, P. Vaccinology: time to change paradigm? Lancet Infect. Dis. (in the press).

5. Aaby, P., Ravn, H. \& Benn, C. S. The WHO review of the possible non-specific effects of diphtheriatetanus-pertussis vaccine. Pediatr. Infect. Dis. J. 35 1247-1257 (2016).

6. Klein, S. L., Shann, F., Moss, W. J., Benn, C. S. \& Aaby, P. RTS,S malaria vaccine and increased mortality in girls. $m B$ Bio 7, e00514-16 (2016).

7. Aaby, P. et al. Testing the hypothesis that diphtheriatetanus-pertussis vaccine has negative non-specific and sex-differential effects on child survival in high-mortality countries. BMJ Open 2, e000707 (2012).

8. Shann, F. A live-vaccine-last schedule: saving an extra million lives a year? Clin. Infect. Dis. https://doi.org/ 10.1093/cid/ciaa292 (2020).

9. Sørup, S. et al. Live vaccine against measles, mumps, and rubella and the risk of hospital admissions for nontargeted infections. JAMA 311, 826-835 (2014).

10. Bardenheier, B. H., McNeil, M. M., Wodi, A. P., McNicholl, J. M. \& DeStefano, F. Risk of nontargeted infectious disease hospitalizations among US children following inactivated and live vaccines, 2005-2014. Clin. Infect. Dis. 65, 729-737 (2017).

11. Freyne, B. \& Curtis, N. Does neonatal BCG vaccination prevent allergic disease in later life? Arch. Dis. Child. 99, 182-184 (2014)

12. Benitez, M. L. R. et al. Mycobacterium bovis BCC in metastatic melanoma therapy. Appl. Microbiol. Biotechnol. 103, 7903-7916 (2019).

13. Guallar-Garrido, S. \& Julian, E. Bacillus CalmetteGuérin (BCG) therapy for bladder cancer: an update. Immunotargets Ther. 9, 1-11 (2020).

14. Netea, M. G. et al. Defining trained immunity and its role in health and disease. Nat. Rev. Immunol. https://doi.org/10.1038/s41577-020-0285-6 (2020).

15. Kühtreiber, W. M. \& Faustman, D. L. BCG therapy for type 1 diabetes: restoration of balanced immunity and metabolism. Trends Endocrinol. Metab. 30, 80-92 (2019).

16. Yamazaki-Nakashimada, M. A. et al. BCG: a vaccine with multiple faces. Hum. Vaccin. Immunother. https://doi.org/10.1080/21645515.2019.1706930 (2020).

17. Gofrit, O. N. et al. Bacillus Calmette-Guérin (BCG) therapy lowers the incidence of Alzheimer's disease in bladder cancer patients. PLoS One 14, e0224433 (2019).

18. de Castro, M. J., Pardo-Seco, J. \& Martinon-Torres, F. Nonspecific (heterologous) protection of neonatal BCC vaccination against hospitalization due to respiratory infection and sepsis. Clin. Infect. Dis. 60, 1611-1619 (2015).

19. Biering-Sorensen, S. et al. Early BCG-Denmark and neonatal mortality among infants weighing $<2500 \mathrm{~g}$ : a randomized controlled trial. Clin. Infect. Dis. 65 , 1183-1190 (2017).

20. Stensballe, L. G. et al. BCG vaccination at birth and early childhood hospitalisation: a randomised clinical multicentre trial. Arch. Dis. Child. 102, 224-231 (2017).

21. Arts, R. J. W. et al. BCG vaccination protects against experimental viral infection in humans through the induction of cytokines associated with trained immunity. Cell Host Microbe 23, 89-100 e105 (2018).

22. Wardhana, Datau, E. A., Sultana, A., Mandang, V. V. $\Sigma \mathrm{Jim}, \mathrm{E}$. The efficacy of bacillus Calmette-Guerin vaccinations for the prevention of acute upper respiratory tract infection in the elderly. Acta Med. Indones. 43, 185-190 (2011).

23. Aaby, P. \& Benn, C. S. Developing the concept of beneficial nonspecific effect of live vaccines with epidemiological studies. Clin. Microbiol. Infect. 25, 1459-1467 (2019).

24. Rieckmann, A. et al. Vaccinations against smallpox and tuberculosis are associated with better long-term survival: a Danish case-cohort study 1971-2010. Int. J. Epidemiol. 46, 695-705 (2017).

25. Klein, S. L., Jedlicka, A. \& Pekosz, A. The Xs and Y of immune responses to viral vaccines. Lancet Infect. Dis. 10, 338-349 (2010)

26. Aaby, P. et al. Long-term survival after EdmonstonZagreb measles vaccination in Guinea-Bissau: increased female mortality rate. J. Pediatr. 122, 904-908 (1993).

27. Aaby, P. et al. Differences in female-male mortality after high-titre measles vaccine and association with subsequent vaccination with diphtheria-tetanuspertussis and inactivated poliovirus: reanalysis of West African studies. Lancet 361, 2183-2188 (2003). 
28. Aaby, P. et al. Increased female-male mortality ratio associated with inactivated polio and diphtheriatetanus-pertussis vaccines: observations from vaccination trials in Guinea-Bissau. Pediatr. Infect. Dis. J. 26, 247-252 (2007).

29. Flanagan, K. L et al. Sex differences in the vaccinespecific and non-targeted effects of vaccines. Vaccine 29, 2349-2354 (2011)

30. Klein, S. L. \& Flanagan, K. L. Sex differences in immune responses. Nat. Rev. Immunol. 16, 626-638 (2016).

31. Flanagan, K. L., Fink, A. L., Plebanski, M. \& Klein, S. L. Sex and gender differences in the outcomes of vaccination over the life course. Annu. Rev. Cell Dev. Biol. 33, 577-599 (2017).

32. Aaby, P. et al. Non-specific effects of standard measles vaccine at 4.5 and 9 months of age on childhood mortality: randomised controlled trial. BMJ 341, c6495 (2010).

33. Benn, C. S., Netea, M. G., Selin, L. K. \& Aaby, P. A small jab - a big effect: nonspecific immunomodulation by vaccines. Trends Immunol. 34, 431-439 (2013)

34. Brook, B. et al. BCG-vaccination induced emergency granulopoiesis provides rapid protection from neonatal sepsis. Sci. Trans/ Med. https://stm. sciencemag.org/lookup/doi/10.1126/scitranslmed. aax4517 (2020).

35. Aaby, P. et al. Measles vaccination in the presence or absence of maternal measles antibody: impact on child survival. Clin. Infect. Dis. 59, 484-492 (2014).

36. Benn, C. S., Fisker, A. B., Whittle, H. C. \& Aaby, P. Revaccination with live attenuated vaccines confer additional beneficial nonspecific effects on overall survival: a review. EBioMed. 10, 312-317 (2016).

37. Kollmann, T. R., Marchant, A. \& Way, S. S. Vaccination strategies to enhance immunity in neonates. Science 368, 612-615 (2020).

38. Noho-Konteh, F. et al. Sex-differential non-vaccinespecific immunological effects of diphtheria-tetanuspertussis and measles vaccination. Clin. Infect. Dis. 63, 1213-1226 (2016)
39. Zivkovic, I. et al. Sexual diergism in antibody response to whole virus trivalent inactivated influenza vaccine in outbred mice. Vaccine $33,5546-5552$ (2015).

40. Zivkovic, I. et al. Sex bias in mouse humoral immune response to influenza vaccine depends on the vaccine type. Biologicals 52, 18-24 (2018).

41. Fink, A. L., Engle, K., Ursin, R. L., Tang, W. Y. $\&$ Klein, S. L. Biological sex affects vaccine efficacy and protection against influenza in mice. Proc. Natl Acad. Sci. USA 115, 12477-12482 (2018).

42. Potluri, T. et al. Age-associated changes in the impact of sex steroids on influenza vaccine responses in males and females. NPJ Vaccines 4, 29 (2019).

43. Andersen, A. et al. National immunization campaigns with oral polio vaccine reduce all-cause mortality: a natural experiment within seven randomized trials. Front. Public Health 6, 13 (2018).

44. Uthayakumar, D. et al. Non-specific effects of vaccines illustrated through the BCG example: from observations to demonstrations. Front. Immunol. 9 , 2869 (2018).

45. Miller, A. et al. Correlation between universal $B C G$ vaccination policy and reduced morbidity and mortality for COVID-19: an epidemiological study. Preprint at medRxiv https://doi.org/10.1101/ 2020.03.24.20042937 (2020)

46. Shet, A. et al. Differential COVID-19-attributable mortality and BCG vaccine use in countries. Preprint at medRxiv https://doi.org/10.1101/2020.04.01. 20049478 (2020).

47. Sala, G. \& Miyakawa, T. Association of BCC vaccination policy with prevalence and mortality of COVID-19. Preprint at medRxiv https://doi.org/ 10.1101/2020.03.30.20048165 (2020).

48. Floc'h, F. \& Werner, G. H. Increased resistance to virus infections of mice inoculated with BCG (bacillus Calmette-Guérin). Ann. Immunol. 127, 173-186 (1976).

49. Curtis, N., Sparrow, A., Ghebreyesus, T. A. $\&$ Neteta, M. G. Considering BCG vaccination to reduce the impact of COVID-19. Lancet https://doi.org 10.1016/S0140-6736(20)31025-4 (2020).
50. Wenham, C., Smith, J. \& Morgan, R. COVID-19: the gendered impacts of the outbreak. Lancet 395 846-848 (2020).

51. Zeng, F. et al. A comparison study of SARS-CoV-2 IgG antibody between male and female COVID-19 patients: a possible reason underlying different outcome between sex. J. Med. Virol. https://doi.org 10.1002/jmv.25989 (2020).

\section{Acknowledgements}

T.R.K. is supported by the NIH National Institute of Allergy and Infectious Diseases (U19AI118608-02) and Telethon Kids and Perth Children's Hospital Foundation. D.J.L's work on the non-specific effects of vaccines is supported by the Flinders Foundation and the Australian National Health and Medical Research Council. The BRACE trial is supported by the Bill \& Melinda Gates Foundation, Sarah and Lachlan Murdoch, the Royal Children's Hospital Foundation, the Minderoo Foundation, the South Australian government, the NAB Foundation, the Calvert-Jones Foundation and individual donors.

\section{Author contributions}

The authors contributed equally to all aspects of the article.

\section{Competing interests}

K.L.F has received consultation and lecture fees from Sanofi Pasteur, Seqirus and Pfizer in the past 5 years and is a member of the Australian Technical Advisory Group on Immunisation. The other authors declare no competing interests.

Publisher's note

Springer Nature remains neutral with regard to jurisdictional claims in published maps and institutional affiliations.

\section{RELATED LINKS}

BRACE trial: https://www.mcri.edu.au/BRACE

Optimmunize consortium: https://www.bandim.org/

optimmunize

Optimmunize meeting, February 2020:

https://coursesandconferences.wellcomegenomecampus.org/

our-events/optimmunize/

(c) Springer Nature Limited 2020 